\title{
Actual time-series of single-cell transcriptomics reveals circadian clocks as key regulators of cell differentiation in Arabidopsis
}

\section{Kotaro Torii}

Kyoto University

Keisuke Inoue

Kyoto University

Keita Bekki

Kyoto University

Kazuya Haraguchi

Otaru University of Commerce

Minoru Kubo

Kumamoto University

Yuki Kondo

Kobe University

Takamasa Suzuki

Chubu University https://orcid.org/0000-0002-1977-0510

Akane Kubota

Nara Institute of Science and Technology

Kyohei Uemoto

Kyoto University

Hanako Shimizu

Kyoto University

Masato Saito

The University of Tokyo

Hiroo Fukuda

Takashi Araki

Kyoto University https://orcid.org/0000-0002-3616-1493

Motomu Endo ( $\nabla$ motomu.endo@gmail.com )

Nara Institute of Science and Technology https://orcid.org/0000-0002-4621-9679

Article

Keywords: circadian clocks, cell differentiation, PeakMacth, LUX, plant stem cells 
Posted Date: July 17th, 2020

DOl: https://doi.org/10.21203/rs.3.rs-40935/v1

License: (c) (i) This work is licensed under a Creative Commons Attribution 4.0 International License. Read Full License 


\section{Abstract}

The basis of toti/pluripotency is elaborate regulation of cell-cycle progression and cell-fate determination. Circadian clocks are involved in this process, but the underlying mechanisms have not been studied due to technical limitations. In particular, there is a lack of research on the universality of cell differentiation mechanisms in multicellular organisms using plants with high cell-fate plasticity. Here, exploiting in vivo single-cell RNA sequencing and a new actual time reconstitution method, PeakMacth, we analyzed actual time-series of cell reprogramming and differentiation processes at single-cell resolution, and found that the circadian clock modulates cell differentiation via BES1-mediated GSK3 signaling, which has a $\beta$ catenin-like function in Arabidopsis. In this pathway, the clock gene $L U X$ in meristematic stem cells directly targets the CYCD and RETINOBLASTOMA-RELATED $(R B R)$ genes, which are commonly involved in cell-cycle progression and cell-fate determination in plants and animals. In addition, the rhythmicity of the circadian clock was associated with cell state, and the establishment of the circadian rhythm preceded cell differentiation. Thus, our study not only reveals the involvement of the circadian clock in the differentiation of plant stem cells but also demonstrates functionally analogous features in the regulatory system of cell differentiation across species.

\section{Main}

The primitive function of the circadian clock was to gate cell-cycle progression and avoid sunlightinduced DNA damage ${ }^{1}$. Perhaps for this reason, the circadian clock of multicellular organisms is involved in both cell-cycle progression and associated cell-fate transitions, which are closely related to toti/pluripotency of cells ${ }^{2,3}$. In mammalian embryonic stem cells, circadian rhythms are not observed during early developmental stages, but they emerge along with cell differentiation; and the circadian rhythms in differentiated cells disappear when they are reprogrammed ${ }^{4-7}$. Consistently, several mutations in clock genes cause abnormal cell differentiation in mammals ${ }^{7,8}$. In Arabidopsis, a circadian clock associated 1; late elongated hypocoty/ double mutant (cca1 lhy) shows an increased number of free-ending vascular bundles, indicating abnormal vascular cell differentiation in the clock mutant ${ }^{9}$. Therefore, elucidating how the circadian clock is involved in stem cell differentiation is crucial for understanding the basis of toti/pluripotency, and can be approached through characterization of gene expression dynamics during reprogramming and differentiation processes at the single-cell resolution.

Single-cell RNA sequencing (scRNA-seq) with unique molecular identifiers (UMI) ${ }^{10}$ and bioinformatics toolkit such as Wishbone ${ }^{11}$ and Seurat ${ }^{12}$ enables us to reconstruct reliable pseudo time trajectory from scRNA-seq datasets. Using these techniques, differentiation process from stem cells has been intensively studied with high spatial resolution. However, single-cell transcriptome analysis at high temporal resolution with an actual time scale remains a challenge. Reprogramming and differentiation of cells usually take a long time, resulting in sparse sampling points, which makes it difficult to analyze transitions in gene expression that are close in time. Furthermore, only developmental trajectory could be analyzed by pseudo time analyses, and the time scale is literally 'pseudo'. This lack of analysis from a 
time perspective delays our understanding of the molecular roles of the circadian clocks on cell differentiation from stem cells and hinders our understanding of the basic mechanisms of toti/pluripotency across species.

Here, we focused on plant cells that exhibit pluripotency and high cell-fate plasticity. By leveraging these properties of plant cells, and by conferring actual time information to pseudo time-series, we analyzed whole cell-differentiation process with high spatiotemporal resolution. We uncovered the molecular basis by which the circadian clock controls plant cell differentiation, and provided a new concept for the functional similarity of signal transduction pathways in the regulation of plant and animal cell differentiation.

\section{Results}

\section{Involvement of plant circadian clocks in cell differentiation process}

To investigate the involvement of the plant circadian clock in cell differentiation, we applied the vascular cell differentiation induction system, VISUAL ${ }^{13,14}$. In this system, vascular cells (including both xylem and phloem cells) are induced from mesophyll cells through the meristematic stem cells and vascular stem cells within 3 days (Fig. 1a and Supplementary Fig. 1a,b). Two clock mutants, cca 1; Ihy, timing of CAB expression 1 (cca1 Ihy toc1) and a knockdown of LUX ARRHYTHMO (LUX) / PHYTOCLOCK 1 and BROTHER OF LUX ARRHYTHMO (also known as NOX) by artificial microRNA (IuX nox) ${ }^{15}$ showed significant defects in vascular cell differentiation (Fig. 1b and Supplementary Fig. 1c), similarly to a loss of function mutant of BRI1-EMS SUPPRESSOR 1 (BES1), which shows severe defects in vascular cell differentiation in VISUAL ${ }^{13}$. We also confirmed that the clock mutants affect development of vascular bundles, guard cells, and root cells in non-VISUAL conditions (Supplementary Fig. 1d-f), suggesting that the plant circadian clock is generally involved in the process of cell differentiation. Our analyses thus uncovered requirement of a functional circadian clocks for plant cell differentiation.

The VISUAL assay consists of two steps, reprogramming of mesophyll cells to meristematic stem cells and differentiation of the meristematic stem cells to vascular cells. To determine which step is regulated by the circadian clocks, we next measured expression levels of cell-type-specific markers. In the wild type (WT), mesophyll cell markers (CAB3 and $\angle H C B 2.1)^{16}$ rapidly disappeared after induction, followed by expression of meristematic stem cell markers (RGF6 and HAM2) ${ }^{17,18}$. Afterward, vascular stem cell markers (TDR and $A N T)^{13,19}$ and vascular cell markers (IRX3 and $V N D 7^{20}$ appeared in succession (Fig. 1c and Supplementary Fig. 1g). Vascular stem cell markers were not fully induced in the cca 1 Ihy toc 1 , Iux nox, and bes 1 mutants, although the reduction of the mesophyll cell markers and the induction of the meristematic stem cell markers in both mutants were comparable to WT (Fig. 1c and Supplementary Fig. $1 \mathrm{~g})$. Overall, marker gene expression revealed that the circadian clock is involved in the step of differentiation rather than reprograming. 


\section{Reconstruction of actual time-series of scRNA-seq data}

Since the expression of the vascular stem cell markers (TDR and ANT) and the vascular cell markers (IRX3 and VND7) overlapped each other (Fig. 1c and Supplementary Fig. 1g), and vascular cell differentiation proceeded gradually, even in the VISUAL assay (Supplementary Fig. 1b), it was still unclear when and how the circadian clock induces cell differentiation. To circumvent such low spatiotemporal resolution of bulk analysis, which can be attributed to the crude averaging of various cell types, we performed time-series of scRNA-seq in the VISUAL. Making protoplasts and/or nuclear isolation is the conventional method to perform scRNA-seq in plants ${ }^{21-23}$; however, the protoplast isolation is timeconsuming and potentially stress-inducing, it is not suitable for time-series analysis of the circadian clocks. We therefore obtained total RNA from single cells using glass capillaries in vivo context ${ }^{24}$ (Supplementary Fig. 2a and Supplementary Videos 1 and 2). 216 single cell samples harvested every $4 \mathrm{~h}$, from $24 \mathrm{~h}$ before induction up to $84 \mathrm{~h}$ after induction were subjected to RNA-seq, and the collected data were normalized together with time-series of cell-population RNA sequencing (cpRNA-seq) data obtained from whole cotyledons (Supplementary Fig. 2b-e and Supplementary Table 1).

To separate xylem and phloem cell lineages, we first applied the Wishbone method, which can order scRNA-seq data with bifurcating developmental trajectories based on an unsupervised clustering ${ }^{11}$. tdistributed Stochastic Neighbor Embedding (t-SNE) of the dataset was represented by a Y-shaped structure, suggesting that the Wishbone can properly reconstruct developmental trajectories of xylem and phloem cells (Fig. 2a). This assertion was further validated by overlaying expression levels of cell-typespecific markers (Fig. 2b and Supplementary Fig. 3a). For simplicity, we focused on the xylem cell lineage and applied the Seurat method ${ }^{12}$ to improve temporal resolution of the pseudo time trajectory. Seurat allowed us to estimate the most likely location of cells by referring to preset expression patterns of celltype-specific markers. Clustering of the data obtained by applying 24 reference genes in Seurat yielded four major clusters presumed to be mesophyll cells, meristematic stem cells, vascular stem cells, and xylem cells (Supplementary Fig. 3b and Supplementary Table 2). This classification was supported by the enriched GO-terms in each cluster (Supplementary Fig. 3c).

Actual time-series with interval scale contains temporal information, whereas ordinal scale-based pseudo time-series lacks temporal information. Thus, time-series analysis of scRNA-seq remains a difficult problem ${ }^{25}$ and few studies have addressed circadian rhythms in single-cell transcriptomes. To overcome this limitation and to reconstruct actual time-series from single-cell transcriptome datasets, we developed PeakMatch method. The basic concept of PeakMatch is that the timing of significant gene expression peaks can be comparable between scRNA-seq data based on pseudo time-series and cpRNA-seq data based on actual time-series (Supplementary Fig. 4a, see Methods for details). By integrating estimated peak times of 2,217 genes, we reconstructed an actual time-series and succeeded in improving segregation of cell-type-specific markers (Fig. 2c, d). The half-value width of the gene expression peaks in the reconstructed actual time-series was narrow compared to that of cpRNA-seq (Fig. 2e), demonstrating that PeakMatch reduces the crude averaging effect and improves temporal resolution. Thus, the 
Wishbone - Seurat - PeakMatch pipeline (Supplementary Fig. 4b) improved spatiotemporal resolution of scRNA-seq data and enabled us to handle actual time-series data at single-cell resolution. Even though the clock genes were not used as PeakMatch references, the expression rhythms of $L H Y$ and CCA1 showed $24 \mathrm{~h}$ of rhythmicity (Fig. 3a and Supplementary Tables 3 and 4).

\section{BES1 suppresses $L H Y$ expression and induce reprogramming}

On the reconstructed actual time-series, periodic oscillations of circadian rhythms were prominent in differentiated cells and ceased in meristematic stem cells in the actual time-series (Fig. 3b), indicating that circadian clock system was dynamically reconstructed during the reprogramming process. To address how the reconstruction of circadian clock system is triggered, we focused on a transcription factor BES1. BES1 plays a pivotal role in the regulation of cell proliferation and cell differentiation ${ }^{26}$, and a bes 1 mutant has been shown to severely inhibit vascular cell differentiation in VISUAL ${ }^{13,27}$ (Fig. 1b). Like $\beta$-catenin in animals, BES1 has an inactive form phosphorylated by GSK3 and an active form that was dephosphorylated ${ }^{28}$. The active form of dephosphorylated BES1 was highly accumulated in the meristematic stem cell state immediately after induction (Fig. 3c). Since chromatin immunoprecipitation (ChIP)-on-chip data of BES1 has shown that BES1 directly suppressed the expression of $L H Y^{29}$, we hypothesized that perturbations to the circadian clock by BES1 trigger circadian clock oscillations that are arrested in the meristematic stem cells. To test the hypothesis, we performed ChIP-qPCR and confirmed that the enrichment of BES1-GFP at the G-box and E-box motifs in the LHY promoter (Fig. 3d). The luciferase activity of $\angle H Y:: L U C$ was decreased by transient co-expression of bes1-D, a gain-offunction mutant protein ${ }^{30}$, indicating that BES1 acts as a repressor of $L H Y$ expression (Fig. 3e). The timing of the accumulation of activated dephosphorylated BES1 was consistent with the timing of the suppression of $\angle H Y$ and $C C A 1$ expressions (Fig. 3f). CCA1 promoter also has BES1 binding site, the G-box and E-box. Overall, BES1 is involved in the reprogramming process through directly modulating $L H Y$ expression. In the bes 1 mutant, the rhythmic expression of $C A B 3$ regulated by LHY remained after differentiation induction, supporting the involvement of BES1 in the reprogramming process (Fig. 1c).

\section{LUX in the meristematic stem cells simultaneously regulates cell-cycle progression and cell differentiation}

The cease of circadian rhythm in stem cells has been similarly observed in mammalian ES/iPS cells $\mathrm{s}^{4-7}$. As reprogramming resulted in loss of mesophyll cell identity, PSEUDO-RESPONSE REGULATOR 5 (PRR5) and $P R R 7$ expression was diminished and only slight expression was detected in vascular stem cells and vascular cells (Fig. 4a). On the other hand, EARLY FLOWERING 4 (ELF4) and LUX, components of the evening complex $(E C)^{31}$, expression was not induced in mesophyll cells but was significantly induced before vascular stem cell state (Fig. 4a). This tendency was also seen in the correlation coefficient between clock genes and differentiation markers (Fig. 4b). LHY, CCA1, PRR5, and PRR7 were highly 
correlated with mesophyll markers, whereas EC components $L U X$ and ELF4 tended to be correlated with meristematic stem cell or vascular stem cell markers. Since LHY regulates the expression of the EC components $L U X$ and $E L F 4^{31,32}$, and the differentiation induction is inhibited in both bes 1 and cca 1 Ihy toc1 mutants (Fig. 1b), the BES1-LHY pathway explains the induction of LUX in the meristematic stem cells. Given that BES1 acts as an integrated hub in plant growth and development ${ }^{26}$, our data do not rule out the possibility that BES1 regulates $L U X$ induction through other pathways.

To test if reconstruction of circadian clock system precedes differentiation from meristematic stem cells, kinetics of GO-term enrichment scores throughout time course was calculated. GO-terms related to DNA methylation and cell cycle, which are involved in cell differentiation, were significantly enriched soon after the induction of ELF 4 and $L U X$. In addition, the reconstruction of circadian clocks occurred more than 24 $\mathrm{h}$ before induction of GO-terms associated with secondary cell wall biosynthesis, which indicates completion of vascular cell differentiation (Fig. 4c).

Predominant expression of LUX and ELF4 in the meristematic stem cells implies specific functions of EC there. To elucidate direct targets of LUX in the meristematic stem cells, we then performed ChIP-seq in the LUX-GFP expressing plants and detected 5,476 peaks (Supplementary Table 5). Among them, genes involved in leading roles in cell-cycle progression and cell differentiation, such as $C Y C D 3 ; 1, R B R$, and $E 2 F C$, were significantly enriched (Fig. $5 a, b$ ). CYCD3; is a D-type of cyclin that controls $\mathrm{G}_{1}-\mathrm{S}$ transition and cell differentiation ${ }^{33}$. RBR also controls epigenetic regulation through DNA methylation during cell differentiation ${ }^{34}$, and E2Fc is reported as a key regulator for xylem differentiation ${ }^{35}$. Consistent with ChIPseq, the lux nox mutant showed altered CYCD3;1 and E2FC expressions (Fig. 5c). Taken together, we concluded that the reconstructed circadian clock simultaneously regulates cell differentiation through fine-tuning of key factors for epigenetic modifications, cell-fate determination, and cell cycle (Fig. 5d).

\section{Discussion}

From actual time-series single-cell analysis, we here revealed that circadian clock was reset during the meristematic stem cell state (Fig. $3 a, b)$. This result explains the continuous phase resetting of CCA1 in the root tip and the rephase of the circadian clocks during the lateral root initiation ${ }^{36,37}$. Also, the expression profile of circadian clocks was changed dynamically during the late meristematic cell state, prior to differentiation from the meristematic stem cell (Fig. 3b). Similarly, changes in the circadian clock gene expression profile in calli have been observed ${ }^{38}$, suggesting that reconstruction of circadian clocks during cell differentiation is universal this phenomenon. These results implies that differences in the expression profiles and features of tissue/organ-specific circadian clocks are established at an early stage of differentiation, and is consistent with the results that the circadian clock regulates the large part of gene expressions, including key factors for cell differentiation ${ }^{39}$. Especially, LUX (probably EC) is highly expressed in the meristematic stem cells and regulates cell differentiation. This approach will further reveal cell-type-specific functions of circadian clocks. For example, TOC1 also play a significant role in 
regulation of cell-cycle progression ${ }^{40}$, and in our analysis TOC1 had a relatively high correlation coefficient score with meristematic and vascular stem cell markers as with ELF4 and LUX (Fig. 4b).

In many multicellular organisms, the circadian clock controls cell division and differentiation ${ }^{41}$. In plants, this study showed that LUX co-regulated both cell-cycle progression and cell-fate determination in meristem cells, achieving coupling between cell differentiation and cell division (endoreduplication). These frameworks may be extended to animals that have independently acquired circadian clock systems during evolution ${ }^{1,42}$. Indeed, cell differentiation mechanism in plants and animals has functionally analogous features. These include (i) nuclear localization of non-phosphorylated forms of BES1 and $\beta$-catenin ${ }^{28}$, (ii) inactivation by GSK3 kinase ${ }^{43}$, (iii) cell differentiation by CYCD / RB1 in animals and CYCD / RBR in plants ${ }^{44}$, and (iv) inverse correlation between circadian clock oscillations and stemness $^{5}$ (Fig. 5d). Convergent evolution of molecular mechanisms is also found in innate immunity ${ }^{45}$, developmental hourglass ${ }^{46}$, photoperiodicity, and circadian clock system ${ }^{1,42}$ of plants and animals. Since the common mechanism is assumed to be deeply related to the essential of its regulatory system, comparative studies of cell differentiation mechanisms in terms of circadian rhythms will provide a new framework for understanding the basis of totipotency/pluripotency.

Overall, our study not only reveals a new role for the circadian clock in the meristematic stem cells in the regulation of plant differentiation, but also provides a new pipeline to enable actual time-series analysis of single-cell transcriptome and concept for understanding toti/pluripotency.

\section{Methods}

\section{Plant material and growth conditions}

All wild type and transgenic lines used here were Arabidopsis thaliana ecotype Columbia-0 (Col-0). Seeds were surface-sterilized and sown on $0.8 \%$ agar plates containing Murashige and Skoog medium with $0.5 \%$ sucrose or liquid media as described previously ${ }^{14}$. Plants were grown under $12 \mathrm{~L} / 12 \mathrm{D}(12 \mathrm{~h}$ light and $12 \mathrm{~h}$ dark, $84 \mu \mathrm{mol} \mathrm{m} \mathrm{m}^{-2} \mathrm{~s}^{-1}$ ) conditions at $22^{\circ} \mathrm{C}$. For induction of ectopic vascular cell differentiation, plants were entrained by $12 \mathrm{~L} / 12 \mathrm{D}$ conditions for 7 days, although the original protocols ${ }^{14}$ called for growth of plants under continuous light (LL) conditions. Bikinin, 2,4-dichlorophenoxyacetic acid (2,4-D), and kinetin were added to the 8-day-old plants at ZTO. cca1-1 Ihy-11 toc1-2 and cca1-1 Ihy-11 were provided by Takafumi Yamashino ${ }^{47}$ (Nagoya University), lux nox and its parental CAB2::LUC were provided by Dmitri A. Nusinow ${ }^{15}$ (Donald Danforth Plant Science Center), LUX::LUX-GFP/ luX-4 was provided by Philip A. Wigge ${ }^{48}$ (University of Cambridge).

\section{Measurement of cell differentiation, cell proliferation, and cell cycles.}


For lignin staining, plants were fixed in acetic ethanol fixative (75\% glacial acetic acid and $25 \%$ ethanol) for 1 day, stained with $20 \%$ phloroglucinol in $99.5 \%$ ethanol and concentrated $\mathrm{HCl}(1: 19, v / v)$ for $1 \mathrm{~h}$, cleared with chloral hydrate/glycerol $/ \mathrm{H}_{2} \mathrm{O}$ mixture $(8 \mathrm{~g}$ of chloral hydrate in $1 \mathrm{~mL}$ of glycerol and $2 \mathrm{~mL}$ of $\mathrm{H}_{2} \mathrm{O}$ ) for $2 \mathrm{~h}$, and observed under a light microscope. For quantification of the vascular cell induction ratio, areas for xylem cells in the cotyledons were calculated using ImageJ.

For measurement of stomatal index, 10-day-old plants grown under 12L/12D conditions were stained with $50 \mathrm{mg} / \mathrm{mL}$ of propidium iodide (PI) and observed using a confocal laser scanning microscope, FV1000 (Olympus) in three square areas of $0.48 \mathrm{~mm}^{2}$ per cotyledon from 5 cotyledons of 5 independent plants. The stomatal index was calculated as previously described ${ }^{49}$. Error bars, representing standard errors, were calculated from the results of 5 independent cotyledons.

For root elongation measurements, plants were grown vertically under 12L/12D conditions. Root length was measured at ZTO. For quantification of root meristem size, 7-day-old plants grown under 12L/12D conditions were stained with $20 \mathrm{mg} / \mathrm{mL} \mathrm{PI}$ and observed using an FV1000 microscope as described above. Meristematic cell numbers were determined from observations of the cortical cells, using confocal microscopy images.

For GUS staining, plants were fixed in $90 \%(\mathrm{v} / \mathrm{v})$ acetone for $15 \mathrm{~min}$ on ice, vacuum-infiltrated and incubated at $37^{\circ} \mathrm{C}$ for $2 \mathrm{~h}$ (overnight for IRX3:: GUS) in the GUS assay solution containing $100 \mathrm{mM}$ sodium phosphate buffer ( $\mathrm{pH} 7.2$ ), $1 \mathrm{mM}$ potassium-ferrocyanide (5 mM for TDR::GUS), $1 \mathrm{mM}$ potassiumferricyanide (5 mM for TDR::GUS), 0.1\%(v/v) Triton X-100 and $0.5 \mathrm{mg} \mathrm{mL}^{-1}$ 5-bromo-4-chloro-3-indolyl- $\beta$ D-glucuronic acid (X-Gluc). Chlorophylls in the tissue were removed by incubation in $70 \%(\mathrm{v} / \mathrm{v})$ ethanol.

\section{qRT-PCR}

Total RNA was extracted using an RNeasy Plant Mini Kit (QIAGEN) and reverse-transcribed using a Transcriptor First Strand cDNA Synthesis Kit (Roche) according to the manufacturer's instructions.

Real-time gene expression was analyzed with a CFX96 Real-Time PCR Detection System (Bio-Rad). $U B Q 14$ were used as an internal control for VISUAL experiments ${ }^{14}$, respectively. Specific sequences for each primer pair were:

CAB3-RT-F, 5'- ACCCAGAGGCTTTCGCGGAGT;

CAB3-RT-R, 5'- TGCGAAGGCCCATGCGTTGT;

LHCB2.1_Fw 5'- ACCCGGAGACATTCGCTAAGAAC;

LHCB2.1_Rv 5'- TGGATCAAGTTAGGGTTTCCGAGG; 
RGF6 Fw 5'- CACAAGGTTGCTTCTTTGGACG;

RGF6 Rv 5'- CACCAACATGGTGAATGCATATGC;

HAM2 Fw 5'- CAAACGGCGGAGATAACAAT;

HAM2 Rv 5'- CTGTGGAACGGAGGTTTAGG;

TDR-RT-F, 5'- TGGTGGAAGTTACTTTGAAGGAG;

TDR-RT-R, 5'- TTCAATCTCTGTAAACCACCGTAA;

ANT_fw, 5'- TCAATACCGAGGCGTTACAAGAC;

ANT_rv, 5'- TCGAGCAGCTTTCTCCTCCATATC;

IRX3-RT-F, 5'- CCTCGGCCACAGCGGAGGAT;

IRX3-RT-R, 5'- CGCCTGCCACTCGAACCAGG;

VND7_fw, 5'- TTCGAAACGCAGTCGTATAATCC;

VND7_rv, 5'- ATTAGCTTCGACCTCATTATAGCTTTG;

CCA1-RT-F, 5'- GAGGCTTTATGGTAGAGCATGGCA;

CCA1-RT-R, 5'- TCAGCCTCTTTCTCTACCTTGGAGA;

LHY-RT-F, 5'- ACGAAACAGGTAAGTGGCGACA;

LHY-RT-R, 5'- TGCGTGGAAATGCCAAGGGT;

CYCD3;1-qPCR-Fw, 5'- CGAAGAATTCGTCAGGCTCT;

CYCD3;1-qPCR-Rv, 5'- ACTTCCACAACCGGCATATC;

E2Fc-qPCR-Fw, 5'-; GAGTCTCC-CACGGTTTCAG;

E2Fc-qPCR-Rv, 5'-; TCACCATCCGGTACTGTTGC;

UBQ14-qPCR-Fw, 5'- TCCGGATCAGGAGAGGTT; and

UBQ14-qPCR-Rv, 5'- TCTGGATGTTGTAGTCAGCAAGA.

The following thermal cycling profile was used,

CAB3, $95^{\circ} \mathrm{C}$ for $10 \mathrm{~s}, \sim 40$ cycles of $95^{\circ} \mathrm{C}$ for $10 \mathrm{~s}, 62^{\circ} \mathrm{C}$ for $15 \mathrm{~s}$, and $72^{\circ} \mathrm{C}$ for $15 \mathrm{~s}$; 
CCA $1,95^{\circ} \mathrm{C}$ for $60 \mathrm{~s}, \sim 40$ cycles of $95^{\circ} \mathrm{C}$ for $10 \mathrm{~s}, 60^{\circ} \mathrm{C}$ for $15 \mathrm{~s}$, and $72^{\circ} \mathrm{C}$ for $7 \mathrm{~s}$;

IRX3, $95^{\circ} \mathrm{C}$ for $60 \mathrm{~s}, \sim 40$ cycles of $95^{\circ} \mathrm{C}$ for $10 \mathrm{~s}, 64.5^{\circ} \mathrm{C}$ for $15 \mathrm{~s}$, and $72^{\circ} \mathrm{C}$ for $10 \mathrm{~s}$;

ANT, VND7, RGF6, and HAM2, $95^{\circ} \mathrm{C}$ for $30 \mathrm{~s}, \sim 40$ cycles of $95^{\circ} \mathrm{C}$ for $5 \mathrm{~s}$ and $60^{\circ} \mathrm{C}$ for $30 \mathrm{~s}$; and

TDR, LHY, CYCD3;1, E2Fc, and UBQ14, $95^{\circ} \mathrm{C}$ for $60 \mathrm{~s}, \sim 40$ cycles of $95^{\circ} \mathrm{C}$ for $10 \mathrm{~s}, 60^{\circ} \mathrm{C}$ for $15 \mathrm{~s}$, and $72^{\circ} \mathrm{C}$ for $15 \mathrm{~s}$.

Each sample was run in technical triplicate to reduce experimental errors. Error bars, representing standard errors, were calculated from the results of biological triplicates.

\section{scRNA-seq and cpRNA-seq}

For scRNA-seq, the process closely followed the method described by Kubo et $a^{R 4}$. A cotyledon before and after treatment with VISUAL for the indicated time periods was placed adaxial side down on a glass slide, and fixed with an adhesive tape, e.g., cellophane tape. Then the center of cotyledon was cut using a razor blade, and with the aid of a microscope, the contents of a single cell were collected using a glass capillary. Samples were subjected to UMI-tagged sequencing using a NextSeq 500 system (Illumina). Detailed methods are described in Supplementary information. For cpRNA-seq, total RNA was extracted using an RNeasy Plant Mini Kit and subjected to UMI-tagged sequencing, as for scRNA-seq, except that 10 cycles of the PCR amplification step were required. Sequence reads were mapped against to the TAIR10 Arabidopsis cDNA sequence by Bowtie ${ }^{50}$ with the parameter "-a --best -strata". Gene expression level was quantified by counting the absolute number of UMls mapped to each gene. scRNA-seq data was normalized together with cpRNA-seq using the R/Bioconductor DESeq ${ }^{51}$ package (http://bioconductor.org/packages/release/bioc/html/DESeq.html; v.1.34.1).

\section{Wishbone - Seurat - PeakMatch pipeline}

To identify scRNA-seq data related to the xylem cell lineage, Wishbone was performed as previously described ${ }^{11}$. Samples after induction in VISUAL were subjected to Wishbone. The first and third components of principal component analysis (PCA) were used for t-SNE. The xylem lineage was selected according to the expression of xylem cell marker genes on the t-SNE plots.

Clustering of cells was performed using the Seurat R package ${ }^{12}$. In brief, digital gene expression matrices were column-normalized and log-transformed. To obtain a landmark gene set for Seurat, we divided all genes in cpRNA-seq data into 17 groups according to the peak expression time of each gene. The 17 genes showing the highest correlation coefficient with scRNA-seq data in each respective group were selected as landmark genes. In addition to the 17 genes, cell-type-specific markers (CAB3, LHCB2.1, TDR, 
AtHB8, IRX3, IRX8, and SEOR1) were also selected as landmark genes. In total, 24 genes were used as a landmark gene set for Seurat.

Finally, we selected the genes whose correlation coefficient between scRNA-seq and cpRNA-seq was more than 0.5. Among the selected genes, 2,217 genes whose sum total of expression levels in the scRNA-seq data were higher than the value equivalent to ten times the cell numbers were subjected to PeakMatch with the following parameters: $T=2$, last $=0$, intv $=1$, inter $=7$.

\section{Overview of the PeakMatch algorithm}

Let be the set of whole genes under consideration. Discretizing pseudo and actual times into integers for simplicity, we denote by and the sets of available pseudo and actual times, respectively. Suppose that, for each gene, we are given pseudo time-series based scRNA-seq data and actual time-series based cpRNA-seq data, where and represent the gene $z$ 's expression levels at a pseudo time $p$ in the scRNA-seq data, and at an actual time $a$ in the cpRNA-seq data, respectively.

To estimate the actual times of gene expressions in the scRNA-seq data, we would like to find pairs of pseudo and actual times so that the expression levels and are likely to be "comparable" for many genes . Once such pairs are found, we may estimate the actual time of by that of .

The point is that, among the observed gene expression levels, "peaks" are the most important phenomena. Then it is desired that a peak in and a peak in should be matched. It is also required that the pseudo time order should be preserved in the time pairs. To be more precise, whenever a pseudo time $p$ is matched to an actual time $a$, any pseudo time $p^{\prime}>p$ should be matched to an actual time $a^{\prime}>a$.

We formulated the problem of finding such time pairs as the maximum weighted non-crossing matching (MWNCM) problem for a bipartite graph. The problem is polynomially solvable ${ }^{52}$, meaning that it is efficiently solvable from the standpoint of the theory of computational complexity.

We took the bipartite graph so that one vertex subset was the pseudo time set and the other vertex subset was the actual time set . For the edge set, we considered all possible pairs, where we determined the weight of an edge heuristically by how the pseudo time $p$ and the actual time $a$ were comparable peaks. We determined the weight of an edge as follows. For each gene, we decided whether or not the value was within a "peak area" in. We considered that was within a peak area if it was significantly larger than a general trend of , which was estimated by an exponential moving average. We set the weight of to a larger value if both and are among peak areas for more genes.

Given the scRNA-seq and cpRNA-seq data, the algorithm PeakMatch constructed the bipartite graph, derived an MWNCM for it, and estimated the actual times of all pseudo times in based on the derived MWNCM. 
For more details and python-based programs, see https://github.com/endo-lab/PeakMatch.

\section{Plasmid construction}

For IRX3::GUS, the promoter of IRX3 was amplified from Col-0 genomic DNA using the following primers:

IRX3p-Fw (Xhol), 5'-cgcggccgcactcgaTCGAGAGCCCGA; and

IRX3p-Rv (Xhol), 5'-gtctagatatctcgaAGGGACGGCCGGAGATTAGCAGCGA.

The amplified fragment was cloned into the Xhol site of pENTR1A (no ccdB). After sequence verification, this plasmid was recombined with pGWB3 ${ }^{53}$, and introduced into Col-0 plants.

For BES1::BES1-GFP, the genomic DNA fragment of BES1 containing the $2 \mathrm{~kb}$ promoter sequence and the $1 \mathrm{~kb}$ downstream sequence from the stop codon was amplified from Col-0 genomic DNA using the following primers:

CACC-pBES1_2k_F, 5'- CACCTCTCAACCTGCTCGGT; and

gBES1-R, 5'- CTCTGATGTGGAGTCAATG.

The amplified fragment was cloned into the pENTR/D-TOPO (Thermo Fisher Scientific). After sequence

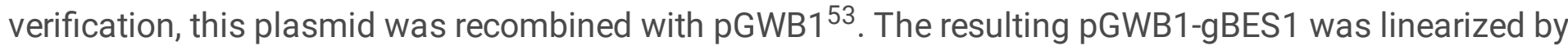
PCR using the following primers:

gBES1-C_inverse-F, 5'- TGAGATGAAGTATACATGAACCTG; and

bes1_R_nonstop, 5'- ACTATGAGCTTTACCATTTCCAAGCG.

Then, sGFP fragment was amplified using the following primers:

sGFP_for-gBES1-C_F, 5'- GGTAAAGCTCATAGTATGGTGAGCAAGGGCG; and

sGFP_for-gBES1-C_R, 5'- GTATACTTCATCTCACTTGTACAGCTCGTCCATG.

The sGFP fragment was inserted just before the stop codon of BES1 by fusion of the two fragments using In-Fusion HD Cloning Kit. After sequence verification, this plasmid was introduced into the bes 1-3 mutant.

For transactivation assay constructs, the NOS terminator was amplified by PCR using the following primers:

nosT-F, 5'-gccgcactcgagatatctagaATCGTTCAAACATTTGGCAA; and 
nosT-R, 5'-tacaagaaagctgggtctagaGATCTAGTAACATAGATGAC.

The amplified fragment was cloned into the Xbal site of pENTR1A (no ccdB) using an In-Fusion HD Cloning Kit (TaKaRa). In the same way, the coding sequence of LUC+ was amplified and cloned into the Xhol-EcoRV site of the plasmid using the following primers:

LUC-Xhol-F, 5'-ttcgcggecgcactcgagATGGAAGACG; and

LUC-EcoRV-R, 5'-tgaacgattctagatatcTTACACGGCGATCTTTCCGC.

The resulting pENTR1A (LUC-nosT) was used for $L H Y:: L U C$. The promoter of $L H Y$ was amplified from Col0 genomic DNA using the following primers:

LHY-pro-F (Xhol), 5'-cgcggccgcactcgaTTTTGGAATAATTTCGGTTATTTC; and

LHY-pro-R (Xhol), 5'-atccgcggatctcgaAACAGGACCGGTGCA.

The amplified fragment was cloned into the Xhol site of pENTR1A (LUC-nosT). The coding sequences of bes 1-D $(L)$ from cDNA of the bes 1-D mutant and RLUC from the vector pRL vector (Promega) were amplified by PCR using the following primers:

bes1-D(L)-F, 5'- GGACTCTAGAGGATCATGAAAAGATTCTTCTATAATTCC;

bes1-D(L)-R, 5'- CGGTACCCGGGGATCTCAACTATGAGCTTTACCATTTCC;

RLUC-F, 5'- GGACTCTAGAGGATCATGACTTCGAAAGTTTATGATCC; and

RLUC-R, 5'-CGGTACCCGGGGATCTTATTGTTCATTTTTGAGAAC.

The amplified fragments were cloned into the BamHI site of pPZP211/NP/35S-nos $T^{54}$ using an In-Fusion HD Cloning Kit.

\section{Western blotting}

Plants expressing BES1::BES1-GFP were harvested every $8 \mathrm{~h}$ from $24 \mathrm{~h}$ before induction in VISUAL, up to $72 \mathrm{~h}$ after induction. Approximately $50 \mathrm{mg}$ of seedlings were ground into a fine powder in liquid nitrogen with a mortar and pestle, mixed with equal volumes of 2' Laemmli sample buffer $(100 \mathrm{mM}$ Tris- $\mathrm{HCl}(\mathrm{pH}$ 6.8), $4 \%(\mathrm{w} / \mathrm{v})$ SDS, $10 \%(\mathrm{v} / \mathrm{v})$ 2-mercaptoethanol, and $20 \%(\mathrm{v} / \mathrm{v})$ glycerol) and boiled at $95^{\circ} \mathrm{C}$ for $5 \mathrm{~min}$. Samples were separated by SDS-PAGE on a 7.5\% acrylamide gel, and transferred onto polyvinylidene fluoride membranes (Bio-Rad Laboratories). For the primary antibody, polyclonal anti-GFP (MBL-598) was diluted 1:2,000. For the secondary antibody, ECL Rabbit IgG, HRP-linked whole Ab (GE Healthcare) was diluted 1:10,000. Blots were visualized with ECL Prime reagent (GE Healthcare) and ImageQuant LAS 4010 (GE Healthcare). 


\section{ChIP-qPCR and ChIP-seq}

Chromatin immunoprecipitation assays using BES1::BES1-GFP/ bes1-3 and LUX::LUX-GFP/ luX-4 were performed as described ${ }^{55}$ with modifications. Briefly, $600 \mathrm{mg}$ of seedlings at $8 \mathrm{~h}$ after induction in VISUAL were fixed in PBS containing 1\% paraformaldehyde for $10 \mathrm{~min}$ at room temperature, and nuclei and chromatin were isolated. The isolated chromatin was sheared with a Covaris S220 sonicator under these parameters: $4-6^{\circ} \mathrm{C}, 175 \mathrm{~W}$ peak power, $5 \%$ duty factor, $200 \mathrm{cycles} / \mathrm{burst}$, for $50 \mathrm{~s}$ of treatment. To immunoprecipitate chromatin, $10 \mu \mathrm{L}$ of anti-GFP antibody (MBL-598) and $50 \mu \mathrm{L}$ of Dynabeads Protein $\mathrm{G}$ (Thermo Fisher Scientific) were used. The precipitated samples were subjected to qPCR or library preparation for ChIP-seq. MYB30 ${ }^{56}$ was used as a positive control for ChIP with BES1. For ChIP-qPCR, specific sequences for each primer pair were:

LHYp-ChIP-1F, 5'- GATTCGGGTAGTTCAGTTCTTCG;

LHYp-ChIP-1R, 5'- GGTTAGTTCGGTTCGGTTCTAGG;

LHYp-ChIP-2F, 5'- CACCGTACCCACTTGTTTAGTCG;

LHYp-ChIP-2R, 5'- CGAGCCAGAAGCTTCAATGTG;

LHYp-ChIP-3F, 5'- GGCTCGTAGAGAAGCAACTTGAG;

LHYp-ChIP-3R, 5'- AGTCATCGCAGATCGACACG;

LHYp-ChIP-4F, 5'- GTGGATTCGTTTGGGTGAGG;

LHYp-ChIP-4R, 5'- AACAGTCGCTGCTTCTCCAG.

MYB30-ChIP-F, 5'-AGGTATTTTACGCTGGAAAATGTGT;

MYB30-ChIP-R, 5'- GAATCATCATAATAAGTATGGAGGTG;

ACT2-ChIP-F, 5'- CGTTTCGCTTTCCTTAGTGTTAGCT; and

ACT2-ChIP-R, 5'- AGCGAACGGATCTAGAGACTCACCTTG.

The following thermal cycling profile was used for all the primers: $95^{\circ} \mathrm{C}$ for $60 \mathrm{~s}, \sim 40$ cycles of $95^{\circ} \mathrm{C}$ for 10 $\mathrm{s}, 60^{\circ} \mathrm{C}$ for $45 \mathrm{~s}$.

For ChIP-seq, the sequence libraries were prepared using a TruSeq ChIP Sample Preparation Kit v2 (Illumina), and sequenced using an Illumina NextSeq 500 system with a 75-nt single-end sequencing protocol. The sequence reads were mapped to the TAIR10 Arabidopsis genome sequence by HISAT2 ${ }^{57}$ 
with default parameters. Peaks were identified by MACS2 ${ }^{58}$, using the matching INPUT control with the genome size parameter "-g 1.3e8".

\section{Transactivation assay}

Agrobacterium cultures carrying plasmids for the transactivation assay were grown overnight at $28^{\circ} \mathrm{C}$, collected by centrifugation, and adjusted to an OD600 of 0.4 with infiltration buffer (10 mM MES(pH 5.6), $10 \mathrm{mM} \mathrm{MgCl}_{2}, 150 \mu \mathrm{M}$ acetosyringone, and $0.02 \%$ Silwet-L77). Cells were kept at $28^{\circ} \mathrm{C}$ in the dark for 1 to $2 \mathrm{~h}$ and then infiltrated into the abaxial air spaces of 4-week-old $N$. benthamiana plants grown under 12L/12D conditions at ZT0. After infiltration, plants were kept under 12L/12D conditions for $36 \mathrm{~h}$ and harvested at ZT12. The transactivation assay was performed with a Dual-Luciferase Reporter Assay System (Promega) according to the manufacturer's instructions.

\section{Data Availability}

Code for PeakMatch is available online at https://github.com/endo-lab/PeakMatch. Other related, relevant lines and data supporting the findings of this study are available from the corresponding authors upon reasonable request.

Sequence data from this article can be found in The Arabidopsis Information Resource (TAIR) databases (https://www.arabidopsis.org) under the following accession numbers: UBQ14 (At4g02890), ACT2 (At3g18780), LHCB2.1 (At2g05100), CAB2 (At1g29920), CAB3 (At1g29910), AtHB8 (At4g32880), TDR (At5g61480), ANT (At4g37750), IRX3 (At5g17420), IRX8 (At5g54690), VND7 (At1 g71930), SEOR1 (At3g01680), COR15A (At2g42540), ADH1 (At1g77120), RD29A (At5g52310), HAM2 (At3g60630), RGF6 (At4g16515), MYB30 (At3g28910), CCA1 (At2g46830), LHY (At1g01060), PRR3 (At5g60100), PRR5 (At5g24470), PRR7 (At5g02810), PRR9 (At2g46790), TOC1 (At5g61380), ELF3 (At2g25930), ELF4 (At2g40080), LUX/PCL1 (At3g46640), BOA/NOX (At5g59570), BES1 (At1g19350), CYCD3;1 (At4g34160), E2Fc (At1g47870), and RBR (At3g12280).

\section{Declarations}

\section{Acknowledgements}

We thank T. Koto, Y. Kanesaka, T. Kondo, and Y. Sando for technical assistance, J.A. Hejna for English proofreading, N. Mochizuki for the gift of CAB2::GUS seeds, T. Demura for the gift of TDR::GUS seeds, T. Nakano for the gift of bes7-D seeds. This work was supported by JST PRESTO grant 888067 (to M.E.); by JSPS KAKENHI (grant numbers 15H05958, 16H01240, 17K19392, 18H04781, and 18H02461 (to M.E.), 19H05674, 19H04866, and 19K16170 ( to A.K), 18K14732 (to K.I.), and 17J08107 (to K.T.)); by ISHIZUE 2017 of the Kyoto University Research Development Program, grants from the Yamada Science 
Foundation, Senri Life Science Foundation, LOTTE Foundation, Daiichi Sankyo Foundation of Life Science, The Takeda Science Foundation, and the Nakajima Foundation, the Sekisui Chemical Grant Program, SEI Group CSR Foundation, SECOM Science and Technology Foundation, Tokyo Kasei Chemical Promotion foundation (to M.E.), The Takeda Science Foundation, Senri Life Science Foundation, and The Mitsubishi Foundation (to A.K.), and ; by Grants-in-Aid for Scientific Research on Priority Area 25113005 (to T.A.). Sample preparation for RNA-seq and ChIP-seq was performed at the Medical Research Support Center, Graduate School of Medicine, Kyoto University.

\section{Author contributions}

K.T., K.I., K.B., H.S., A.K., and K.U. performed the gene expression analysis and phenotypic analysis. K.H. and K.T. performed the bioinformatic analysis. T.S., M.K., K.T., H.S., and M.E. performed the single-cell RNA-seq analysis. K.I. performed the ChIP assay. Y.K. assisted with VISUAL experiments. M.S. established BES1::BES1-GFP plants. K.T., K.I., and M.E. wrote the manuscript. M.E. directed and supervised the research with the support of T.A. and H.F..

\section{Competing interests}

The authors declare no competing interests.

\section{References}

\section{References}

1. Hut, R. A. \& Beersma, D. G. Evolution of time-keeping mechanisms: early emergence and adaptation to photoperiod. Trans. R Soc. Lond. B Biol. Sci. 366, 2141-2154 (2011).

2. Weger, M., Diotel, N., Dorsemans, A. C., Dickmeis, T. \& Weger, B. D. Stem cells and the circadian clock. Biol. 431, 111-123 (2017).

3. Tsuchiya, Y., Umemura, Y. \& Yagita, K. Circadian clock and cancer: From a viewpoint of cellular differentiation. J. Urol. 27, 518-524 (2020).

4. Kowalska, E., Moriggi, E., Bauer, C., Dibner., C. \& Brown, S. A. The circadian clock starts ticking at a developmentally early stage. Biol. Rhythms 25, 442-449 (2010).

5. Yagita, K. et al. Development of the circadian oscillator during differentiation of mouse embryonic stem cells in vitro. Natl. Acad. Sci. U S A 107, 3846-3851 (2010).

6. Paulose, J. K., Rucker, E. B. \& Cassone, V. M. Toward the beginning of time: circadian rhythms in metabolism precede rhythms in clock gene expression in mouse embryonic stem cells. PLOS ONE 7, e49555 (2012). 
7. Dierickx, P., Van Laake, L. W. \& Geijsen, N. Circadian clocks: from stem cells to tissue homeostasis and regeneration. EMBO Rep. 19, 18-28 (2018).

8. Yu, X. et al. TH17 cell differentiation is regulated by the circadian clock. Science $\mathbf{3 4 2 , 7 2 7 - 7 3 0}$ (2013).

9. Aihara, K., Naramoto, S., Hara, M. \& Mizoguchi, T. Increase in vascular pattern complexity caused by mutations in $\angle H Y$ and $C C A 1$ in Arabidopsis thaliana under continuous light. Plant Biotechnol. 31, 43-47 (2014).

10. Islam, S. et al. Quantitative single-cell RNA-seq with unique molecular identifiers. Methods 11, 163166 (2014).

11. Setty, M. et al. Wishbone identifies bifurcating developmental trajectories from single-cell data. Biotechnol. 34, 637-645 (2016).

12. Satija, R., Farrell, J. A., Gennert, D., Schier, A. F. \& Regev, A. Spatial reconstruction of single-cell gene expression data. Biotechnol. 33, 495-502 (2015).

13. Kondo, Y. et al. Plant GSK3 proteins regulate xylem cell differentiation downstream of TDIF-TDR signalling. Commun. 5, 3504 (2014).

14. Kondo, Y. et al. Vascular Cell Induction Culture System Using Arabidopsis Leaves (VISUAL) reveals the sequential differentiation of sieve element-like cells. Plant Cell 28, 1250-1262 (2016).

15. Helfer, A. et al. LUX ARRHYTHMO encodes a nighttime repressor of circadian gene expression in the Arabidopsis core clock. Biol. 21, 126-133 (2011).

16. Endo, M., Shimizu, H., Nohales, M. A., Araki, T. \& Kay, S. A. Tissue-specific clocks in Arabidopsis show asymmetric coupling. Nature 515, 419-422 (2014).

17. Matsuzaki, Y., Ogawa-Ohnishi, M., Mori, A. \& Matsubayashi, Y. Secreted peptide signals required for maintenance of root stem cell niche in Arabidopsis. Science 329, 1065-1067 (2010).

18. Engstrom, E. M. et al. Arabidopsis homologs of the petunia hairy meristem gene are required for maintenance of shoot and root indeterminacy. Plant Physiol. 155, 735-750 (2011).

19. Campbell, L. \& Turner, S. Regulation of vascular cell division. Exp. Bot. 68, 27-43 (2017).

20. Yamaguchi, M. et al. VASCULAR-RELATED NAC-DOMAIN7 directly regulates the expression of a broad range of genes for xylem vessel formation. Plant J. 66, 579-590 (2011).

21. Shulse, C. N. et al. High-throughput single-cell transcriptome profiling of plant cell types. Cell Rep. 27, 2241-2247.e4 (2019).

22. Ryu, K. H., Huang, L., Kang, H. M. \& Schiefelbein, J. Single-cell RNA sequencing resolves molecular relationships among individual plant cells. Plant Physiol. 179, 1444-1456 (2019).

23. Nelms, B. \& Walbot, V. Defining the developmental program leading to meiosis in maize. Science $\mathbf{3 6 4}$, 52-56 (2019).

24. Kubo, M. et al. Single-cell transcriptome analysis of Physcomitrella leaf cells during reprogramming using microcapillary manipulation. Nucleic Acids Res. 47, 4539-4553 (2019). 
25. Sun, N. et al. Inference of differentiation time for single cell transcriptomes using cell population reference data. Commun. 8, 1856 (2017).

26. Li, Q. F. et al. The brassinosteroid-regulated transcription factors BZR1/BES1 function as a coordinator in multisignal-regulated plant growth. Biochim Biophys Acta Gene Regul Mech. 1861, 561-571 (2018).

27. Saito, M., Kondo, Y. \& Fukuda, H. (2018). BES1 and BZR1 redundantly promote phloem and xylem differentiation. Plant Cell Physiol. 259, 590-600.

28. Sharma, M., Pandey, A. \& Pandey, G. K. $\beta$-catenin in plants and animals: common players but different pathways. Front Plant Sci. 5, 143 (2014).

29. Yu, X. et al. A brassinosteroid transcriptional network revealed by genome-wide identification of BESI target genes in Arabidopsis thaliana. Plant J. 65, 634-646 (2011).

30. Yin, Y. et al. BES1 accumulates in the nucleus in response to brassinosteroids to regulate gene expression and promote stem elongation. Cell 109, 181-191 (2002).

31. Nusinow, D. A. et al. The ELF4-ELF3-LUX complex links the circadian clock to diurnal control of hypocotyl growth. Nature 475, 398-402 (2011).

32. Li, G. et al. Coordinated transcriptional regulation underlying the circadian clock in Arabidopsis. Cell Biol. 13, 616-622 (2011).

33. Collins, C., Maruthi, N. M. \& Jahn, C. E. CYCD3 D-type cyclins regulate cambial cell proliferation and secondary growth in Arabidopsis. Exp. Bot. 66, 4595-4606 (2015).

34. Kuwabara, A. \& Gruissem, W. Arabidopsis RETINOBLASTOMA-RELATED and Polycomb group proteins: cooperation during plant cell differentiation and development. Exp. Bot. 65, 2667-2676 (2014).

35. Taylor-Teeples, M. et al. An Arabidopsis gene regulatory network for secondary cell wall synthesis. Nature 517, 571-575 (2015).

36. Fukuda, H., Ukai, K. \& Oyama, T. Self-arrangement of cellular circadian rhythms through phaseresetting in plant roots. Rev. E Stat. Nonlin. Soft Matter Phys. 86, 041917 (2012).

37. Voß, U. et al. The circadian clock rephases during lateral root organ initiation in Arabidopsis thaliana. Commun. 6, 7641 (2015).

38. Lee, K., Park, O. S., \& Seo, P. J. RNA-Seq analysis of the Arabidopsis transcriptome in pluripotent calli. Cells 39, 484-94 (2016).

39. Michael, T. P. et al. Network discovery pipeline elucidates conserved time-of-day-specific cisregulatory modules. PLoS Genet. 4, e14 (2008).

40. Fung-Uceda, J. et al. The circadian clock sets the time of DNA replication licensing to regulate growth in Dev. Cell 45, 101-113.e4. (2018).

41. Brown S. A. Circadian clock-mediated control of stem cell division and differentiation: beyond night and day. Development 141, 3105-3111 (2014). 
42. Bhadra, U., Thakkar, N., Das, P. \& Pal Bhadra, M. Evolution of circadian rhythms: from bacteria to human. Sleep Med. 35, 49-61 (2017).

43. Youn, J. H. \& Kim, T. W. Functional insights of plant GSK3-like kinases: multi-taskers in diverse cellular signal transduction pathways. Plant 8, 552-565 (2015).

44. Desvoyes, B., de Mendoza, A., Ruiz-Trillo, I. \& Gutierrez, C. Novel roles of plant RETINOBLASTOMARELATED (RBR) protein in cell proliferation and asymmetric cell division. Exp. Bot. 65, 2657-2666 (2014).

45. Mermigka, G., Amprazi, M., Mentzelopoulou, A., Amartolou, A. \& Sarris, P. F. Plant and animal innate immunity complexes: fighting different enemies with similar weapons. Trends Plant Sci. 25, 80-91 (2020).

46. Quint, M. et al. A transcriptomic hourglass in plant embryogenesis. Nature 490, 98-101 (2012).

\section{References for Methods}

47. Niwa, Y. et al. Genetic linkages of the circadian clock-associated genes, TOC1, CCA1 and $L H Y$, in the photoperiodic control of flowering time in Arabidopsis thaliana. Plant Cell Physiol. 48, 925-937 (2007).

48. Ezer, D. et al. The evening complex coordinates environmental and endogenous signals in Arabidopsis. Nat. Plants 3, 17087 (2017).

49. Kang, C. Y., Lian, H. L., Wang, F. F., Huang, J. R. \& Yang, H. Q. Cryptochromes, phytochromes, and COP1 regulate light-controlled stomatal development in Arabidopsis. Plant Cell 21, 2624-2641 (2009).

50. Langmead B., Trapnell C., Pop M. \& Salzberg S. L. Ultrafast and memory-efficient alignment of short DNA sequences to the human genome. Genome Biol. 10, R25.

51. Anders, S \& Huber, W. Differential expression analysis for sequence count data. Genome Biol. 11, R106 (2010).

52. Malucelli, F., Ottmann, T. \& Pretolani, D. Efficient labelling algorithms for the maximum noncrossing matching problem. Discrete Appl. Math. 47, 175-179 (1993).

53. Nakagawa, T. et al. Development of series of gateway binary vectors, pGWBs, for realizing efficient construction of fusion genes for plant transformation. Biosci. Bioeng. 104, 34-41 (2007).

54. Nishimura, T., Yokota, E., Wada, T., Shimmen, T. \& Okada, K. An Arabidopsis ACT2 dominant-negative mutation, which disturbs F-actin polymerization, reveals its distinctive function in root development. Plant Cell Physiol. 44, 1131-1140 (2003).

55. Yamaguchi, N. et al. PROTOCOLS: Chromatin immunoprecipitation from Arabidopsis Arabidopsis Book 12, e0170, doi:10.1199/tab.0170 (2014).

56. Li, L. et al. Arabidopsis MYB30 is a direct target of BES1 and cooperates with BES1 to regulate brassinosteroid-induced gene expression. Plant J. 58, 275-286 (2009). 
57. Kim, D., Langmead, B. \& Salzberg, SL. HISAT: a fast spliced aligner with low memory requirements. Methods 12,357-360 (2015).

58. Zhang, Y. et al. Model-based analysis of ChIP-Seq (MACS). Genome Biol. 9, R137 (2008).

\section{Supplementary Tables And Videos}

Supplementary tables and videos were not provided with this version.

\section{Supplementary Table 1.}

Summary of single-cell transcriptome data.

\section{Supplementary Table 2.}

List of a landmark gene set for Seurat.

\section{Supplementary Table 3.}

Reconstructed actual time-series of single-cell transcriptome data using the Wishbone - Seurat PeakMatch pipeline.

\section{Supplementary Table 4.}

Peak expression times of clock genes in the reconstructed actual time.

\section{Supplementary Table 5.}

Summary of peak calling using the MACS2 and gene annotation of putative LUX target genes.

Supplementary Video 1. Extracting a single-cell content using a glass capillary. Part 1.

Supplementary Video 2. Extracting a single-cell content using a glass capillary. Part 2.

\section{Figures}



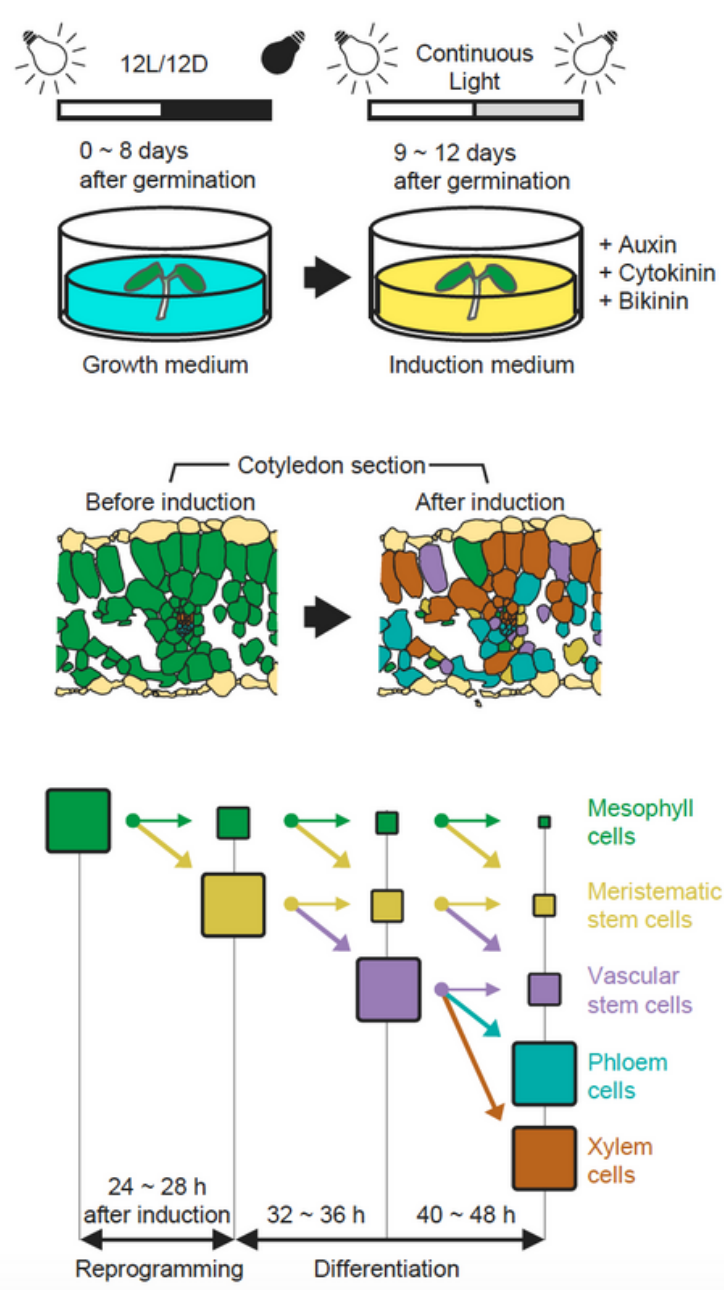

b

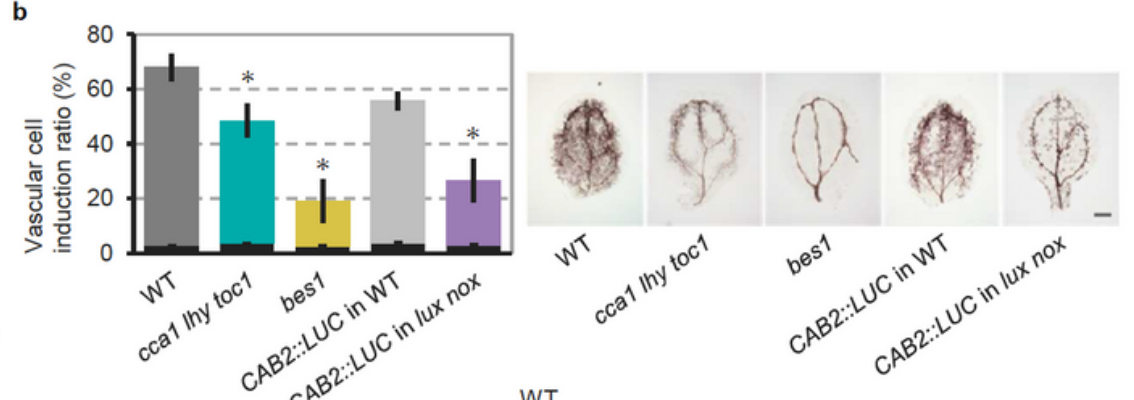

c

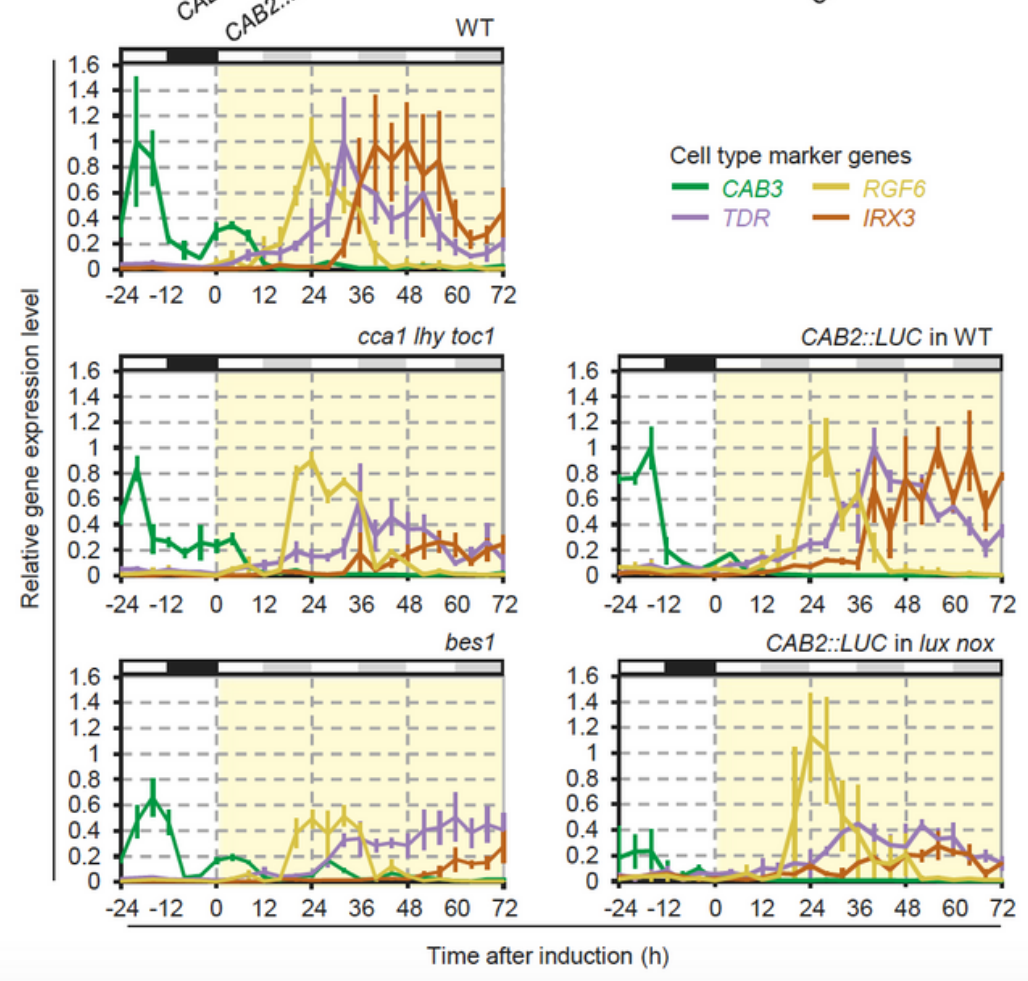

Figure 1

The plant circadian clock affects cell differentiation. a, Schematic of ectopic vascular cell induction with VISUAL. The size of the square schematically represents the proportion of cells. b, Lignin stained xylem cell density in WT, bes 1 , and clock mutants $96 \mathrm{~h}$ after induction. Gray and colored bars indicate ectopically induced xylem cells, and black bars indicate endogenous xylem cells (left, $n=5$, mean \pm s.e., Dunnett's test, ${ }^{*} p<0.05$ ). Representative photos of lignin staining (right, bar $=0.5 \mathrm{~mm}$ ). $c$, Expression patterns of cell-type-specific markers during VISUAL in WT, cca1 lhy toc1, lux nox, and bes1 ( $n=3$, mean \pm s.e.). Green, yellow, purple, and red lines indicate marker genes for mesophyll cells (CAB3), meristematic stem cells (RGF6), vascular stem cells (TDR), and xylem cells (IRX3), respectively. Expression peaks of each respective gene in WT were normalized to 1 . White, black, and gray boxes indicate light period, night period, and subjective night period, respectively. 

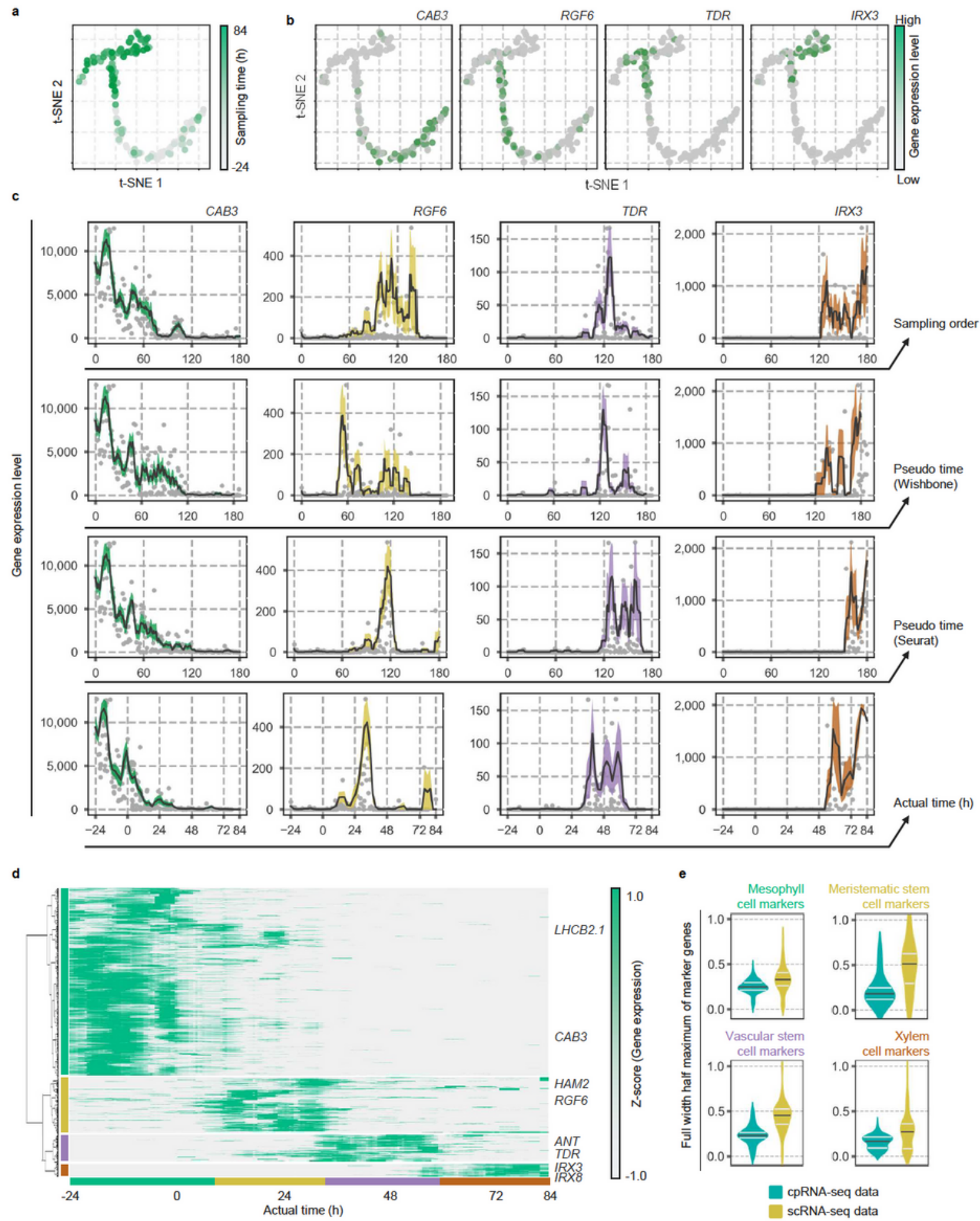

Figure 2

Wishbone - Seurat - PeakMatch pipeline improves temporal resolution of scRNA-seq data. $a, b$, Bifurcated cell lineage on t-SNE 2D plots predicted by Wishbone, showing actual time of sampling (sampling time), and overlaid by expression levels of cell-type-specific markers. Color codes indicate sampling times from early (gray) to late (green) (a) and normalized UMI counts from low (gray) to high (green) (b). c, Comparison of marker gene expression patterns aligned by sampling order, pseudo time 
(Wishbone), pseudo time (Seurat) (Wishbone and Seurat were applied), and actual time (Wishbone Seurat - PeakMatch were applied). Black solid lines are moving average of 3 cells before and after (for sampling order, pseudo time (Wishbone), and pseudo time (Seurat)) and 2 hours before and after (for actual time). Colored clouds are moving standard errors. Gray circles represent the single-cell data. d, A hierarchically-clustered heat map visualizing Z-scores of moving averages of gene expression levels with a window size of $4 \mathrm{~h}$. Green, yellow, purple, and red bars indicate marker genes for mesophyll cells, meristematic stem cells, vascular stem cells, and xylem cells, respectively. e, Comparison of full width at half maximum of cell-type-specific marker expression peaks between the actual time-series scRNA-seq data and the cpRNA-seq data. Black lines indicate the median. White lines indicate the upper and lower quartiles. 
a

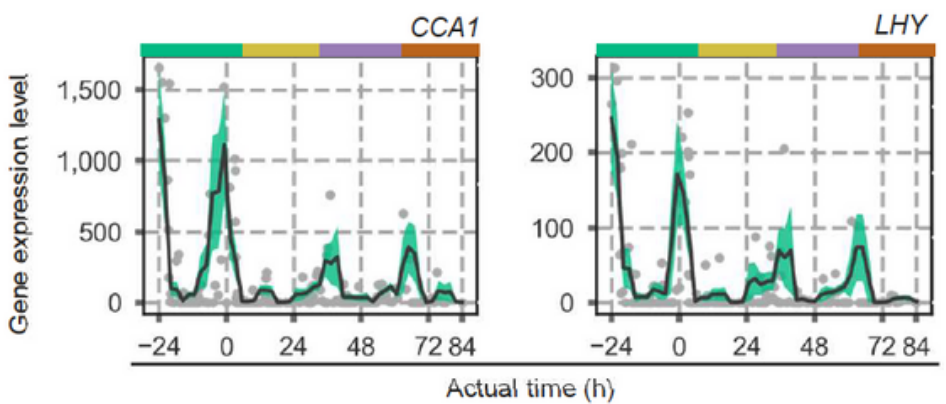

b

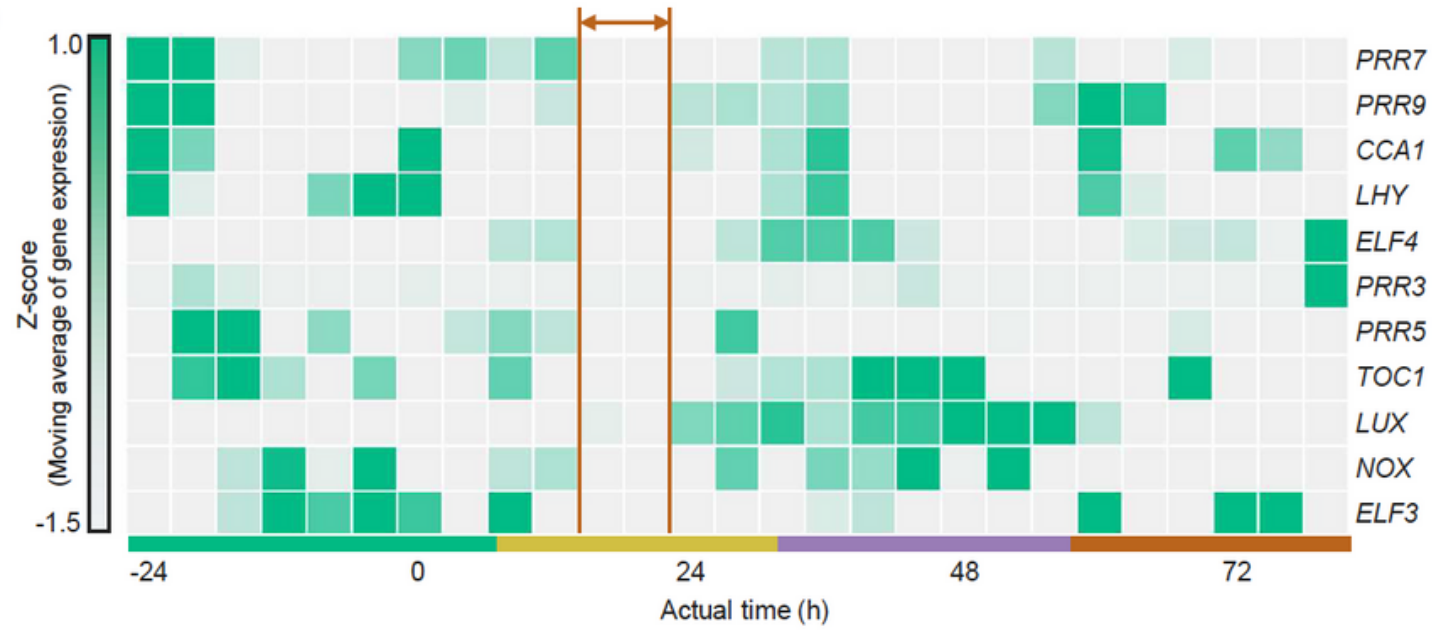

c

Time after induction $(\mathrm{h})$
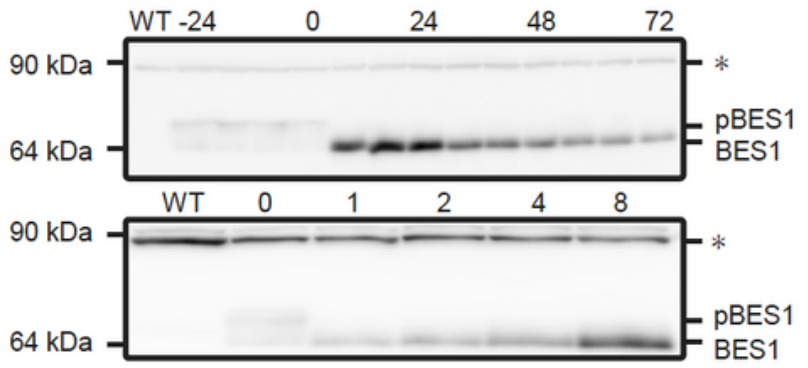

e

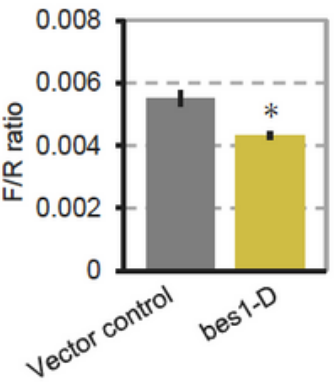

d

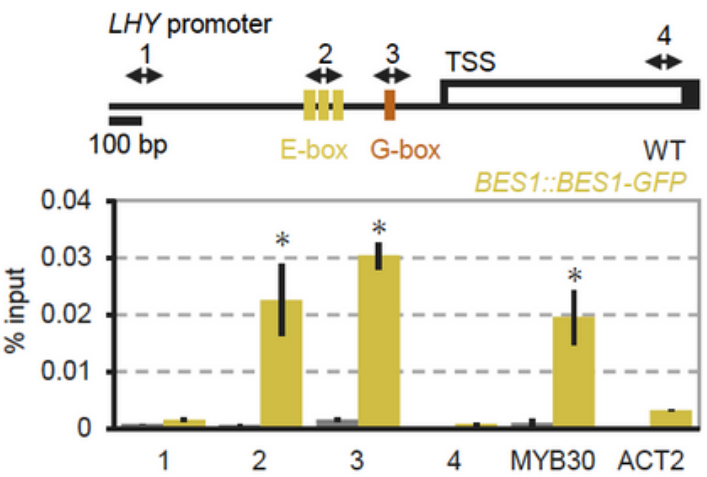

f

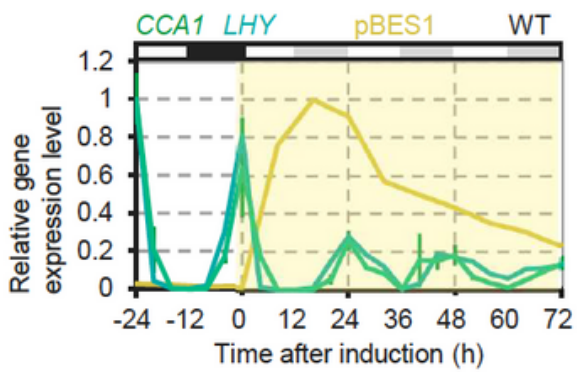

Figure 3

The circadian clock is reconstructed during reprogramming. a, Reconstruction of $24 \mathrm{~h}$ periodicity in timeseries scRNA-seq by the Wishbone - Seurat - PeakMatch pipeline. Green, yellow, purple, and red bars indicate mesophyll cell, meristematic stem cell, vascular stem cell, and xylem cell states, respectively. Black solid lines are moving average of 2 hours before and after. Green clouds are moving standard errors. Gray circles represent the single-cell data. b, A heat map visualizing Z-scores of moving averages 
of each clock gene expression level and their sum total with window size of $4 \mathrm{~h}$. A double-headed arrow indicates a period of time when clock genes cease their rhythmic expression in the meristematic stem cell states. Green, yellow, red, and blue bars indicate periods of time corresponding to mesophyll cell, meristematic stem cell, vascular stem cell, and xylem cell states, respectively. c, Immunoblot analyses of BES1-GFP proteins during VISUAL. Samples were harvested every $8 \mathrm{~h}$, from $24 \mathrm{~h}$ before induction up to $72 \mathrm{~h}$ after induction (top) and from 1 to $8 \mathrm{~h}$ after induction (bottom). pBES1 indicates phosphorylated BES1. Asterisks indicate non-specific bands. $d$, ChIP-qPCR analyses using WT and BES1::BES1-GFP ( $n=$ 3 , mean \pm s.e., two-sided Student's t-test with Bonferroni correction, $p<0.05)$. MYB30 and ACT2 were used as positive and negative controls, respectively. Yellow and orange boxes indicate E-box (CANNTG) and G-box (CACGTG) motifs. TSS: transcriptional start site. e, Expression of LHY::LUC using 35S::bes1-D as an effector in N. benthamiana ( $n=5$, mean \pm s.e., two-sided Student's t-test, $p<0.05)$. 35S::RLUC was used as a transformation control. F/R ratio indicates ratio of firefly and Renilla luciferase activities. $f$, Expression patterns of LHY and CCA1 during VISUAL $(n=3$, mean \pm s.e.). Relative dephosphorylated BES1 levels determined from Fig. $3 \mathrm{c}$ are overlaid in yellow. 

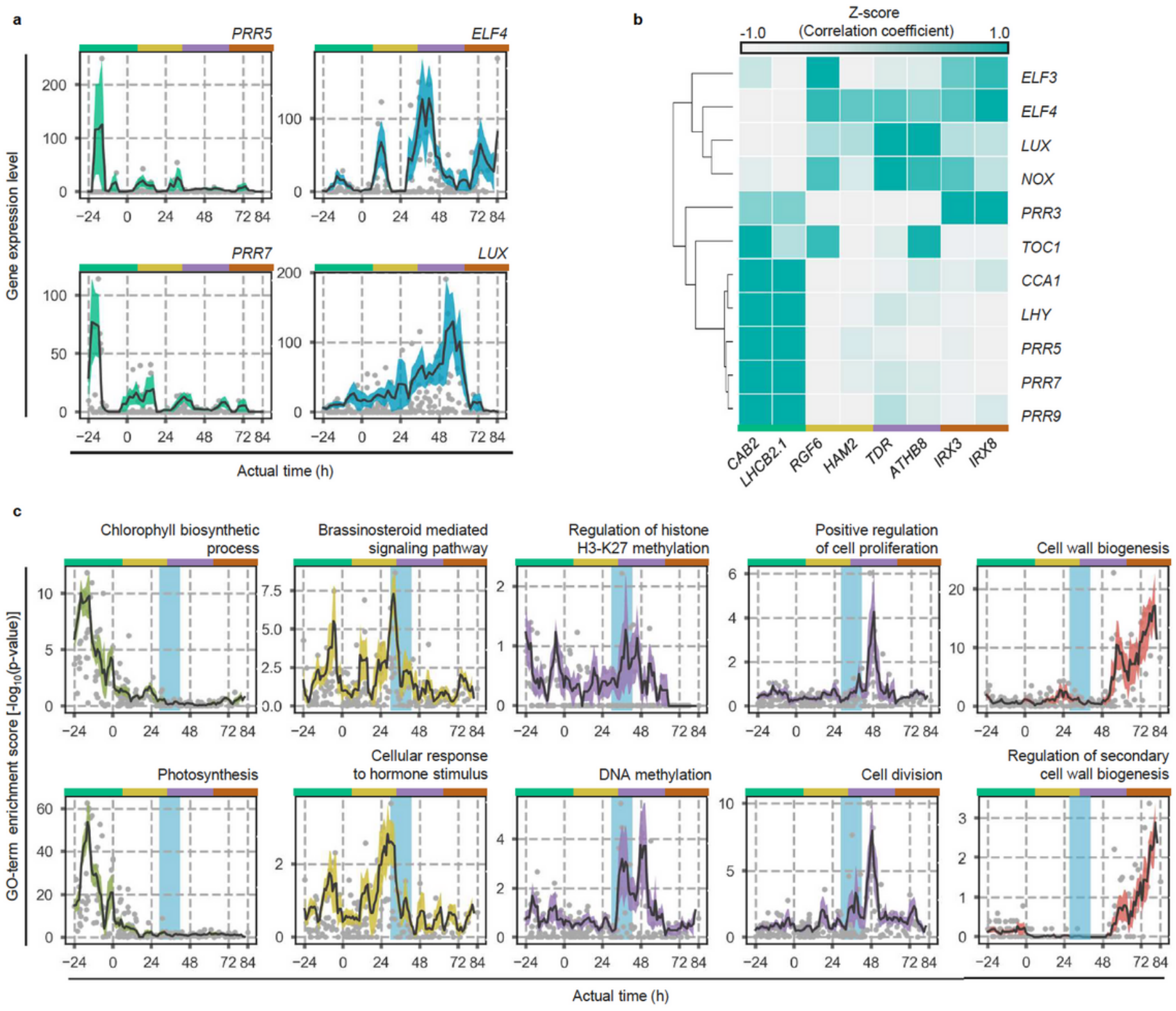

\section{Figure 4}

Reconstruction of the circadian clock precedes cell differentiation. a, Expression patterns of clock genes in actual time-series scRNA-seq data. Expression of ELF4 and LUX originates in the meristematic stem cell. Green, yellow, purple, and red bars indicate mesophyll cell, meristematic stem cell, vascular stem cell, and xylem cell states, respectively. Black solid lines are moving average of 2 hours before and after. Colored clouds are moving standard errors. Gray circles represent the single-cell data. b, Pearson's correlation coefficient between clock genes and cell-type-specific markers. c, GO-term enrichment during cell-fate transition. The first peak time of ELF4 and LUX expression is highlighted by the pale blue window. Green, yellow, purple, and red bars indicate mesophyll cell, meristematic stem cell, vascular stem cell, and xylem cell states, respectively. Black solid lines are moving average of 2 hours before and after. Colored clouds are moving standard errors. Gray circles represent the single-cell data. 
a

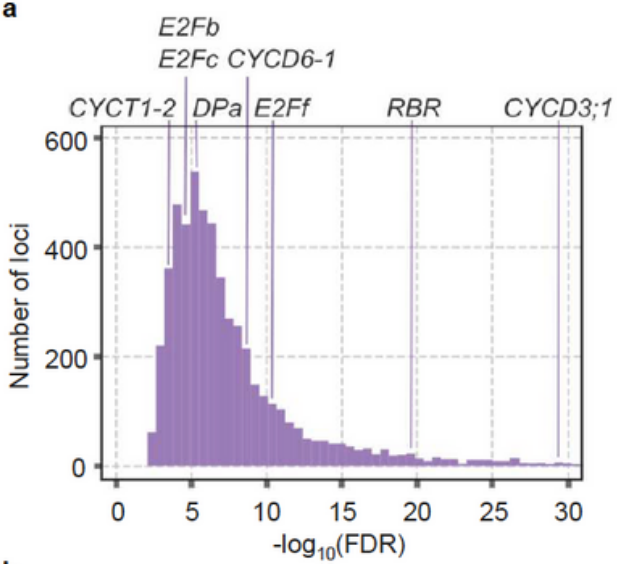

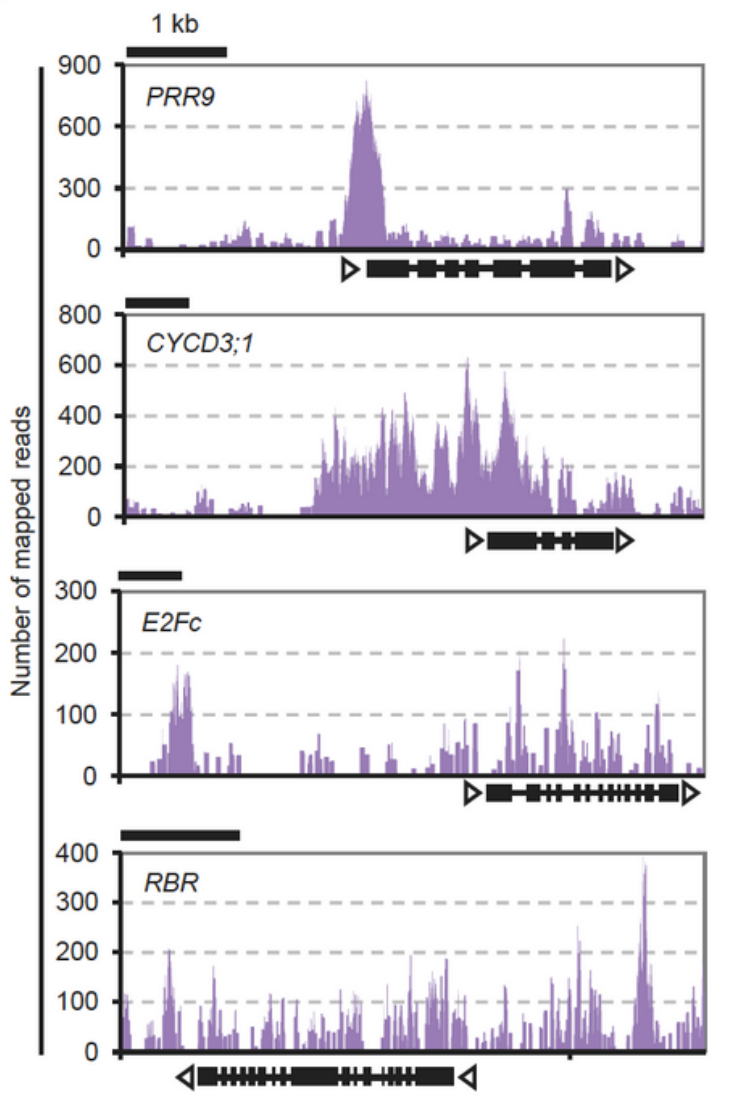

d

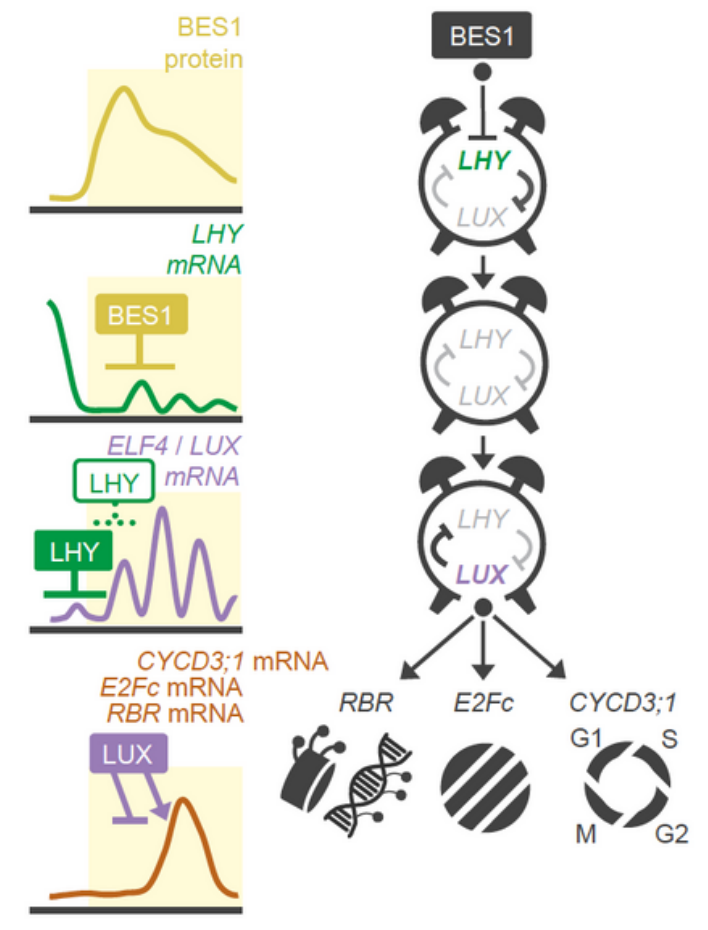

Figure 5

LUX regulates cell-cycle progression and cell differentiation. a, FDR distributions of genes related to G1-S transition in the candidate LUX target genes. b, Visualization of ChIP-seq data around genes related to clock, cell cycle, cell-fate determination, and epigenetic regulation (bar $=1 \mathrm{~kb}$ ). Peak counts of reads are shown. c, Expression patterns of CYCD3;1 and E2Fc during VISUAL in lux nox and corresponding WT ( $\mathrm{n}=$ 3 , mean \pm s.e.). Peak expression levels of each respective gene in WT were normalized to 1 . White, black, and gray boxes indicate light period, night period, and subjective night period, respectively. $d$, Our model proposes that BES1 represses LHY expression in meristematic stem cells to reconstruct the circadian clock, resulting in phase resetting and restart of the clock with dynamic changes of clock genes 
expression. LUX in the reconstructed clock modulates key factors for epigenetic regulation, cell-fate determination, and cell cycle, thereby inducing cell differentiation.

\section{Supplementary Files}

This is a list of supplementary files associated with this preprint. Click to download.

- ToriiSupplementarylnformation.doc

- ToriiSupplementaryFigure1.pdf

- ToriiSupplementaryFigure2.pdf

- ToriiSupplementaryFigure3.pdf

- ToriiSupplementaryFigure4.pdf 